\title{
Candida Colonization among Neonates with Low Birth Weight: There Is Much More to Explore
}

\author{
Wilhelmina Mussa1, Benard Modest², Adolfine Hokororo1, Florentina Mashuda1, Vitus Silago², \\ Stephen E. Mshana2 ${ }^{2}$, Martha F. Mushi2 ${ }^{*}$
}

${ }^{1}$ Department of Pediatrics and Child Health, Weill Bugando School of Medicine, Catholic University of Health and Allied Sciences, Mwanza, Tanzania

${ }^{2}$ Department of Microbiology and Immunology, Weill Bugando School of Medicine, Catholic University of Health and Allied Sciences, Mwanza, Tanzania

Email: *mushimartha@gmail.com

How to cite this paper: Mussa, W., Modest, B., Hokororo, A., Mashuda, F., Silago, V., Mshana, S.E. and Mushi, M.F. (2020) Candida Colonization among Neonates with Low Birth Weight: There Is Much More to Explore. Open Journal of Pediatrics, $10,626-634$.

https://doi.org/10.4236/ojped.2020.104064

Received: September 11, 2020

Accepted: November 27, 2020

Published: November 30, 2020

Copyright $\odot 2020$ by author(s) and Scientific Research Publishing Inc. This work is licensed under the Creative Commons Attribution International License (CC BY 4.0).

http://creativecommons.org/licenses/by/4.0/

\begin{abstract}
Background and Objectives: Candida colonization in neonates with low birth weight is reported to be the first step for invasive candidiasis. This study was designed to describe the patterns of Candida spp. colonizing neonates with low birth weight in Mwanza, Tanzania to provide baseline information for future studies in this field. Methodology: The hospital based cross sectional study was conducted between January 2019 and April 2019 among neonates with low birth weight. Clinical and social demographic data were collected using interview guide questionnaire. Oral and umbilical rectal swabs were collected on the first and seventh day of life to assess Candida spp. colonization status. Data were analyzed using STATA version 13 following the study objectives. Results: A total of 320 neonates with median body weight of 1600 [IQR 1000 - 2000] grams were enrolled. Prevalence of Candida spp. colonization on either oral, umbilical or rectum was $16.2 \%$ (52/320). A total of $34(10.6 \%)$ and $41(12.8 \%)$ neonates were colonized by Candida spp. on the first day and seventh day of life, respectively. Of 34 neonates colonized on the first day of life, $23(67.7 \%)$ were still being colonized on the seventh day. Candida albicans 38 (73.1\%), was the predominant species detected followed by Candida glabrata 10 (19.2\%). Conclusion: The prevalence of Candida spp. colonization among neonates with low birth weight is within the reported range and is mainly due to Candida albicans. Future studies are highly needed to explore the role of colonization and subsequent Candida spp. infections.
\end{abstract}




\section{Keywords}

Candida Colonization, C. albicans, C. glabrata, Oral

\section{Introduction}

Globally the leading cause of neonatal deaths is infection with $10 \%$ reported to be due to Candida spp. [1] [2]. The mortality due to neonatal-candidiasis is estimated to range between $30 \%$ and $73 \%$ [2] [3]. Candida colonization in neonates with low birth weight is reported to be the first step for invasive candidiasis [2] [4]. Studies have shown 7\% - 25\% of low birth weight neonates are colonized by Candida spp. and are at high risk of developing invasive candidiasis [5] [6]. Among neonates with very low birth weight admitted in intensive care unit, Candida spp. is reported to be the third leading causative agent of sepsis [1] [7].

Neonatal Candida spp. colonization is reported to commonly occur in the first weeks of life whereby up to $10 \%-64 \%$ of neonates can be colonized [8]. The neonatal Candida spp. colonization was reported in a study conducted in India to be significantly more in neonates with low birth weight $<1500$ gram with prevalence of $33.3 \%$ compared to the prevalence of $10 \%$ among neonates with birth weight $>1500$ gram [9].

Risk factors for Candida spp. colonization include: use of broad spectrum antibiotics, resuscitation after birth and per vaginal deliver with colonized mother [9] [10]. Furthermore, Candida spp. colonization has been pronounced more in premature neonates regardless of their birth weight [11]. Studies have documented 7\% - 28.3\% of Candida spp. colonized premature neonates developed blood candidiasis [9] [11] [12]. Invasive procedures like mechanical ventilation, cannulation, central catheterization and parenteral nutrition have been documented as factors associated with invasive Candida spp. infection among colonized neonates with low birth weight [10] [13] [14].

Despite having $40 \%$ - 50\% admissions of neonates with low birth weight at Bugando Medical Centre neonatal intensive care unit (BMC NICU), there is paucity of data on the magnitude and patterns of Candida spp. colonization in this and other centers in the developing countries. Here, we report the prevalence of Candida spp. and their distribution among recruited neonates from intensive care unit of the Bugando Medical Centre (BMC), Sekou Toure Regional Referral Hospital (SRRH) and Sengerema District Designated Hospital (SDDH).

\section{Material and Methods}

The descriptive cross sectional hospital based study was conducted from January 2019 to April 2019. The study recruited neonate with low birth weight below 2500 grams on their first day of life admitted in neonatal intensive care units and premature units of BMC, SRRH and SDDH. The neonatal admission per month at BMC ranges from $100-200$ with about $40 \%-50 \%$ having low birth weight. At 
SRRH and SDDH the admissions due to low birth weight range from 40 - 50 and 150 - 200, respectively. The study excluded all neonates with abdominal wall malformations such as gastroschisis, omphalocele and genital urinary tract like bladder exstrophy. The sample size was obtained by the use of Kish Leslie formula [15] using the prevalence of Candida spp. colonization obtained from a study conducted in Uganda [11].

Screening of the neonatal gestation age and maturity was done using Ballard score [16]. Parents with neonates fulfilling inclusion criteria were interviewed to obtain social demographic and clinical data like age, sex, residence mode of delivery, serological HIV status and history of premature rupture of membrane (PROM). Clinical examination to assess temperature, respiratory rate, presence or absence of cyanosis, jaundice, umbilical redness, convulsions, reduced movements and inability to feed was done by qualified pediatrician. Oral and umbilical-rectal swabs were taken on day 1 and day 7. All swabs were transported to microbiology laboratory in the Stuart transport media (HiMedia-Mumbai, India) within two hours of collection for processing. All swabs were cultured on Sabouraud's dextrose agar (SDA) supplemented with $50 \mu \mathrm{g} / \mathrm{ml}$ gentamicin and $50 \mu \mathrm{g} / \mathrm{ml}$ chloramphenicol (HiMedia-Mumbai, India) as previously described [17]. Colonial morphology, germ tube test and reaction on the chromogenic agar (Brilliance Candida agar, Oxoid-UK) were used for identification of Candida spp.

Data were entered on excel spread sheet for consistent check and cleaning then transferred to STATA version 13 for analysis. Categorical data were summarized using proportions while continuous data were summarized using median and interquartile range. The protocol of this study was ethically approved by the joint CUHAS/BMC research ethics and review committee (CREC) with certificate number CREC /333/2019. Parents were informed about the purpose, procedures, risks and benefits of the participation, prior to obtain written informed consent.

\section{Results}

\subsection{Social Demographic and Clinical Data of 320 Studied Neonates}

A total of 227 (70.8\%), 57 (17.8) and $36(11.4 \%)$ neonates were recruited from BMC, SRRH and SDDH, respectively. Their median body weight was 1600 [IQR 1000 - 2000] grams and median gestation age at delivery being 33 [30 - 35] weeks. Nearly half of neonates were admitted in the neonatal intensive care unit $165(51.6 \%)$. The majority $208(65.0 \%)$ of studied neonates were spontaneous vaginal delivery. A total 231 (72.2\%) of parents resided in urban areas and the slightly majority of studied neonates were female 182 (56.8\%) (Table 1).

The majority of neonates were delivered in the health care facilities 302 (94.4\%) and $192(60.0 \%)$ were resuscitated at birth. A total of $210(65 \%)$ neonates had poor feeding and 194 (65.6\%) had IV cannulation. Antibiotics use was 
Table 1. Socio-demographic distribution and other characteristics of 320 neonates with low birth weight.

\begin{tabular}{|c|c|c|}
\hline Characteristics & Number (n)/Median & Percent (\%)/IQR \\
\hline Body weight (gram) & 1600 & $1000-2000$ \\
\hline GANBS (weeks) & 33 & $30-35$ \\
\hline \multicolumn{3}{|l|}{ Gender } \\
\hline Male & 138 & 43.2 \\
\hline Female & 182 & 56.8 \\
\hline \multicolumn{3}{|l|}{ Ward } \\
\hline Premature & 155 & 48.4 \\
\hline NICU & 165 & 51.6 \\
\hline \multicolumn{3}{|l|}{ Antibiotic use } \\
\hline Yes & 126 & 39.4 \\
\hline No & 194 & 60.4 \\
\hline \multicolumn{3}{|l|}{ Location } \\
\hline Urban & 231 & 72.2 \\
\hline Rural & 89 & 27.8 \\
\hline \multicolumn{3}{|c|}{ Education level of the mother } \\
\hline No formal education & 72 & 22.5 \\
\hline Primary & 135 & 42.2 \\
\hline Secondary & 98 & 30.6 \\
\hline University/college & 15 & 4.7 \\
\hline \multicolumn{3}{|l|}{ Work of the mother } \\
\hline Employed & 47 & 14.7 \\
\hline Self employed & 192 & 60.0 \\
\hline Student & 4 & 1.2 \\
\hline Unemployed & 77 & 24.1 \\
\hline \multicolumn{3}{|c|}{ Frequency of antenatal visit } \\
\hline None & 7 & 2.2 \\
\hline Once & 68 & 21.2 \\
\hline Twice & 100 & 31.2 \\
\hline Thrice & 84 & 26.3 \\
\hline Fourth and above & 61 & 19.1 \\
\hline \multicolumn{3}{|c|}{ Trimester during antenatal booking } \\
\hline First trimester & 92 & 28.8 \\
\hline Second trimester & 216 & 67.5 \\
\hline Third trimester & 12 & 3.7 \\
\hline \multicolumn{3}{|l|}{ Mode of delivery } \\
\hline $\mathrm{C} /$ section & 109 & 34.1 \\
\hline SVD & 208 & 65.0 \\
\hline Ass. Vagina delivery & 3 & 0.9 \\
\hline
\end{tabular}

GANBS is gestation age at delivery by new Ballard score, SVD is spontaneous vertex delivery. 
recorded in about one third of the neonates 126 (39.4\%). Fever (axillary temperature above $37.5^{\circ} \mathrm{C}$ ) was observed in $40(12.5 \%)$ neonates.

\subsection{Candida Spp. Colonization}

A total of $52(16.3 \%)$ neonates were colonized by Candida spp. on both day 1 and 7 . A total of $34(10.6 \%)$ and 41 (12.8\%) neonates were colonized by Candida spp. on first day and seventh day of life, respectively. Of these, 28 (82.4\%) were orally and $6(17.6 \%)$ were colonized on the umbilicus/rectum. Of 34 neonates colonized on the first day of life, 23 (67.7\%) were still being colonized on the seventh day. The majority of neonates were colonized by Candida albicans 38 (73.1\%). Of 14 non-albicans Candida spp. detected 10 (19.2\%) were Candida glabrata, Figure 1.

Of 28 orally colonized neonates, 20 (71.4\%) had Candida albicans. For the second swab taken on day 7 , a total of 41 neonates were colonized and of them $31(75.6 \%)$ were orally colonized. Of 31 neonates colonized orally by Candida spp. 25 (80.6\%) had Candida albicans.

\section{Discussion}

Candida colonization in neonates has been documented as a major predisposing factor to invasive candidiasis [18] [19] [20]. In the current study the prevalence of neonatal candida colonization on either oral, umbilicus or rectum was found to be $16.3 \%$. This prevalence is within the range of $12.4 \%-18 \%$ previously reported in Jordanian, Brazil and India [9] [19] [20] [21]. Horizontal transmission from the nursing mother, overcrowd and sharing of incubators are among risk factors which have previously documented to influence Candida spp. colonization among neonates with low birth weight [9] [10] [11] [13] [14] [22].

The prevalence reported in the current study is significantly lower $(p=0.022)$ than the prevalence reported from Uganda 23.3\% [11]. The study in Uganda recruited much younger neonates with low birth weight than the current study, age and birth weight have been found to increase risk of Candida spp. colonization [11]. In the current study the median gestation age and birth weight was 33 [30 - 35] weeks and 1600 [100 - 200] grams, respectively.

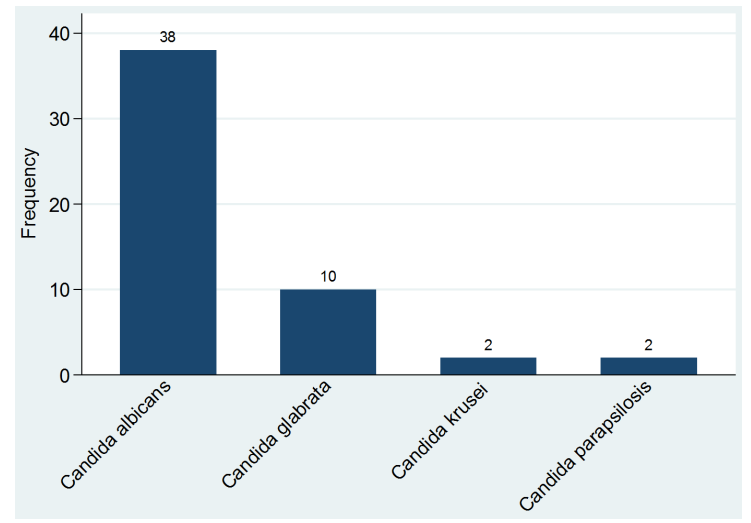

Figure 1. Candida species colonizing low birth weight neonates. 
As in previous studies, [11] [19] [23] Candida albicans was the predominant specie detected. The ability of $C$. albicans to maintain synergistic relationship with other bacteria flora of the skin and buccal cavity can explain the findings [24] [25]. Non-albicans Candida spp. were detected to colonize $26.9 \%$ of colonized neonates with low birth weight. Candida colonization by non-albicans Candida spp. have also been observed in different population from our previous studies [17] [23]. This has also been reported in other studies worldwide in the past decade [26] [27] and is highly associated with increased rate of candida infections due to non-albicans Candidaspp.

Candida glabrata has been noted as one of the leading non-albicans Candida spp. colonizing and causing infections in different population [17] [27]. This has also been observed in the current study whereby Candida glabrata was the most frequently non-albicans Candida spp. isolated. The isolation of Candida glabrata is of public health concern due to its variable susceptibility to fluconazole the most frequently used antifungal agents [28]. Candida glabrata has also been reported as emerging health threat causing neonatal sepsis [27].

\section{Conclusion}

The prevalence of candida colonization among neonates with low birth weight is within the reported range of other studies from Africa, and is mainly due to Candida albicans. Future studies are highly needed to explore the role of colonization and subsequent Candida spp. infections.

\section{Limitation}

The patterns of Candida spp. colonizing these neonates might not reflect the true picture due to low discriminatory index of the techniques used to speciate the isolated Candida spp.

\section{Acknowledgements}

Authors would like to acknowledge the support provided by Department of Microbiology and Immunology of the Catholic University of Health and Allied Sciences, Mwanza, Tanzania.

\section{Ethics Approval and Consent to Participate}

The protocol of this study was ethically approved by the joint CUHAS/BMC research ethics and review committee (CREC) with certificate number CREC/333/2019. All parents of the neonates were requested to sign the written informed consent before recruitment was done. All data were treated as confidential

\section{Consent for Publication}

None applicable. 


\section{Availability of Data and Materials}

The datasets used and/or analyzed during the current study available from the corresponding author on reasonable request.

\section{Conflicts of Interest}

None declared.

\section{Authors' Contributions}

WM and MFM designed the work. WM, FM \& AH recruited patients, BM, VS and MFM performed laboratory investigations and results interpretations. MFM and WM analyzed and interpreted the data. MFM wrote the first draft of the manuscript which was critically reviewed by all authors. All authors read and approved the final version of the manuscript.

\section{Conflicts of Interest}

The authors declare no conflicts of interest regarding the publication of this paper.

\section{References}

[1] Stoll, B.J., Hansen, N., Fanaroff, A.A., Wright, L.L., Carlo, W.A., Ehrenkranz, R.A., Lemons, J.A., Donovan, E.F., Stark, A.R. and Tyson, J.E. (2002) Late-Onset Sepsis in Very Low Birth Weight Neonates: The Experience of the NICHD Neonatal Research Network. Pediatrics, 110, 285-291. https://doi.org/10.1542/peds.110.2.285

[2] Benjamin, D.K., Stoll, B.J., Fanaroff, A.A., McDonald, S.A., Oh, W., Higgins, R.D., Duara, S., Poole, K., Laptook, A. and Goldberg, R. (2006) Neonatal Candidiasis among Extremely Low Birth Weight Infants: Risk Factors, Mortality Rates, and Neurodevelopmental Outcomes at 18 to 22 Months. Pediatrics, 117, 84-92. https://doi.org/10.1542/peds.2004-2292

[3] Baley, J.E. (1991) Neonatal Candidiasis: The Current Challenge. Clinics in Perinatology, 18, 263-280. https://doi.org/10.1016/S0095-5108(18)30523-2

[4] Manzoni, P., Farina, D., Leonessa, M., d’Oulx, E.A., Galletto, P., Mostert, M., Miniero, R. and Gomirato, G. (2006) Risk Factors for Progression to Invasive Fungal Infection in Preterm Neonates with Fungal Colonization. Pediatrics, 118, 2359-2364. https://doi.org/10.1542/peds.2006-1311

[5] Baley, J.E., Kliegman, R.M., Boxerbaum, B. and Fanaroft, A.A. (1986) Fungal Colonization in the Very Low Birth Weight Infant. Pediatrics, 78, 225-232.

[6] Abdallah, Y. (2009) Early Candida Colonization of Preterm Neonates Admitted to Special Care Unit Mulago Hospital, Prevalence, Associated Factors and Early Outcome.

[7] Samaga, M.P. (2017) Prevalence of Neonatal Septicaemia in a Tertiary Care Hospital in Mandya, Karnataka, India. International Journal of Research in Medical Sciences, 4, 2812-2816. https://doi.org/10.18203/2320-6012.ijrms20161956

[8] Berenguer, J., Buck, M., Witebsky, F., Stock, F., Pizzo, P.A. and Walsh, T.J. (1993) Lysis-Centrifugation Blood Cultures in the Detection of Tissue-Proven Invasive Candidiasis Disseminated versus Single-Organ Infection. Diagnostic Microbiology 
and Infectious Disease, 17, 103-109. https://doi.org/10.1016/0732-8893(93)90020-8

[9] Mendiratta, D., Rawat, V., Thamke, D., Chaturvedi, P., Chhabra, S. and Narang, P. (2006) Candida Colonization in Preterm Babies Admitted to Neonatal Intensive Care Unit in the Rural Setting. Indian Journal of Medical Microbiology, 24, 263. https://doi.org/10.4103/0255-0857.29384

[10] Ezenwa, B.N., Oladele, R.O., Akintan, P.E., Fajolu, I.B., Oshun, P.O., Oduyebo, O.O. and Ezeaka, V.C. (2017) Invasive Candidiasis in a Neonatal Intensive Care Unit in Lagos, Nigeria. Nigerian Postgraduate Medical Journal, 24, 150. https://doi.org/10.4103/npmj.npmj_104_17

[11] Abdallah, Y., Kaddu-Mulindwa, D., Nankunda, J. and Musoke, P.M. (2015) Prevalence and Immediate Outcome of Candida Colonized Preterm Neonates Admitted to Special Care Unit of Mulago Hospital, Kampala Uganda. African Health Sciences, 15, 197-205. https://doi.org/10.4314/ahs.v15i1.26

[12] Huang, Y.-C., Li, C.-C., Lin, T.-Y., Lien, R.-I., Chou, Y.-H., Wu, J.-L. and Hsueh, C. (1998) Association of Fungal Colonization and Invasive Disease in Very Low Birth Weight Infants. The Pediatric Infectious Disease Journal, 17, 819-822.

https://doi.org/10.1097/00006454-199809000-00014

[13] Caggiano, G., Lovero, G., De Giglio, O., Barbuti, G., Montagna, O., Laforgia, N. and Montagna, M.T. (2017) Candidemia in the Neonatal Intensive Care Unit: A Retrospective, Observational Survey and Analysis of Literature Data. BioMed Research International, 2017, Article ID: 7901763. https://doi.org/10.1155/2017/7901763

[14] Saiman, L., Ludington, E., Dawson, J.D., Patterson, J.E., Rangel-Frausto, S., Wiblin, R.T., Blumberg, H.M., Pfaller, M., Rinaldi, M. and Edwards, J.E. (2001) Risk Factors for Candida Species Colonization of Neonatal Intensive Care Unit Patients. The Pediatric Infectious Disease Journal, 20, 1119-1124. https://doi.org/10.1097/00006454-200112000-00005

[15] Bourdillon, R., Clarke, C., Ridges, A.P., Sheppard, P., Harper, P. and Leslie, S.A. (1965) "Pink Spot" in the Urine of Schizophrenics. Nature, 208, 453-455. https://doi.org/10.1038/208453a0

[16] Marín, M.G., Martín, J.M., Lliteras, G.F., Delgado, S.G., Pallás, C.A. and Pérez, E.E. (2006) Assessment of the New Ballard Score to Estimate Gestational Age. Anales de Pediatria (Barcelona, Spain: 2003), 64, 140-145.

[17] Mushi, M.F., Mtemisika, C.I., Bader, O., Bii, C., Mirambo, M.M., Groß, U. and Mshana, S.E. (2016) High Oral Carriage of Non-Albicans Candida spp. among HIV-Infected Individuals. International Journal of Infectious Diseases, 49, 185-188. https://doi.org/10.1016/j.ijid.2016.07.001

[18] Smith, P.B., Steinbach, W.J. and Benjamin, J.D. (2005) Neonatal Candidiasis. Infectious Disease Clinics of North America, 19, 603-615.

https://doi.org/10.1016/j.idc.2005.05.007

[19] Issa, S.Y., Badran, E.F., Akl, K.F. and Shehabi, A.A. (2011) Epidemiological Characteristics of Candida Species Colonizing Oral and Rectal Sites of Jordanian Infants. BMC Pediatrics, 11, 79. https://doi.org/10.1186/1471-2431-11-79

[20] Borges, R.M., Soares, L.R., de Brito, C.S., de Brito, D.V.D., Abdallah, V.O.S. and Gontijo, F.P.P. (2009) Risk Factors Associated with Colonization by Candida spp. in Neonates Hospitalized in a Neonatal Intensive Care Unit in Brazil. Revista da Sociedade Brasileira de Medicina Tropical, 42, 431-435. https://doi.org/10.1590/S0037-86822009000400014

[21] Manzoni, P., Stolfi, I., Messner, H., Cattani, S., Laforgia, N., Romeo, M.G., Bollani, L., Rinaldi, M., Gallo, E. and Quercia, M. (2012) Bovine Lactoferrin Prevents Inva- 
sive Fungal Infections in Very Low Birth Weight Infants: A Randomized Controlled Trial. Pediatrics, 129, 116-123. https://doi.org/10.1542/peds.2011-0279

[22] Bliss, J.M., Basavegowda, K.P., Watson, W.J., Sheikh, A.U. and Ryan, R.M. (2008) Vertical and Horizontal Transmission of Candida Albicans in Very Low Birth Weight Infants Using DNA Fingerprinting Techniques. The Pediatric Infectious Disease Journal, 27, 231-235. https://doi.org/10.1097/INF.0b013e31815bb69d

[23] Mushi, M.F., Bader, O., Taverne-Ghadwal, L., Bii, C., Groß, U. and Mshana, S.E. (2017) Oral Candidiasis among African Human Immunodeficiency Virus-Infected Individuals: 10 Years of Systematic Review and Meta-Analysis from Sub-Saharan Africa. Journal of Oral Microbiology, 9, Article ID: 1317579. https://doi.org/10.1080/20002297.2017.1317579

[24] Budtz-Jørgensen, E. (2000) Ecology of Candida-Associated Denture Stomatitis. Microbial Ecology in Health and Disease, 12, 170-185.

https://doi.org/10.1080/089106000750051846

[25] Odds, F. (1984) Ecology and Epidemiology of Candida Species. Zentralblatt für Bakteriologie, Mikrobiologie und Hygiene. 1. Abt. Originale. A, Medizinische Mikrobiologie, Infektionskrankheiten und Parasitologie, 257, 207-212. https://doi.org/10.1016/S0174-3031(84)80073-6

[26] Singhi, S., Rao, D.S.R. and Chakrabarti, A. (2008) Candida Colonization and Candidemia in a Pediatric Intensive Care Unit. Pediatric Critical Care Medicine, 9, 91-95. https://doi.org/10.1097/01.PCC.0000298643.48547.83

[27] Baradkar, V., Mathur, M., Kumar, S. and Rathi, M. (2008) Candida Glabrata: Emerging Pathogen in Neonatal Sepsis. Annals of Tropical Medicine and Public Health, 1, 5. https://doi.org/10.4103/1755-6783.43070

[28] Mushi, M.F., Bader, O., Bii, C., Groß, U. and Mshana, S.E. (2019) Virulence and Susceptibility Patterns of Clinical Candida Sisolates from a Tertiary Hospital, Tanzania. Medical Mycology, 57, 566-572. https://doi.org/10.1093/mmy/myy107 